Research Article

\title{
Environmental Efficiency Evaluation of Chinese Industry Systems by Using Non-Cooperative Two-Stage DEA Model
}

\author{
Xiao Shi ii \\ School of Finance, Shandong University of Finance and Economics, Jinan, Shandong Province 250000, China \\ Correspondence should be addressed to Xiao Shi; shixiao@sdufe.edu.cn
}

Received 3 July 2019; Accepted 17 September 2019; Published 9 October 2019

Academic Editor: Antonios Tsourdos

Copyright (C) 2019 Xiao Shi. This is an open access article distributed under the Creative Commons Attribution License, which permits unrestricted use, distribution, and reproduction in any medium, provided the original work is properly cited.

In evaluating the environmental efficiency analysis of Chinese industry systems, data envelopment analysis (DEA) has been a popular method. However, the production system is often treated as a black box in conventional DEA models. This study considers the internal structure of the production system to evaluate the environmental efficiency, which is characterized as a twostage system, i.e., production subsystem and pollutant treatment subsystem. And, in reality, some subsystems in two-stage production systems are not equally important, and this kind of two-stage systems usually has the feature that one subsystem dominates the other. Thus, we consider the leader and follower relationship in the environmental efficiency analysis. A new noncooperative two-stage DEA model considering undesirable intermediates and undesirable outputs is proposed to calculate the environmental efficiency. The proposed method is then applied to 30 regional industry systems of China in the year 2010. Thus, each DMU's environmental efficiencies for the overall system as well as both subsystems could be analyzed by the proposed approach. More accurate information could be provided for environmental management.

\section{Introduction}

As the policy of "opening and reforms" in 1978, China has achieved tremendous success in economic development. Its economic growth maintains high speed, but its environment deteriorates as many industrial pollutants are discharged to environment [1]. These environmental problems are mainly due to huge pollutants from industries. The government and people have realized that more efforts should be concentrated on protecting the environment. Thus, the Chinese government has paid much attention to the discharge of waste gas, waste water, and solid waste from industry $[2,3]$. However, the environmental issues, such as resource shortage and environmental pollution, are still the problems China faces [4-6]. Environmental issues have attracted many attentions, and environmental efficiency analysis has become more critical.

In previous literature, data envelopment analysis (DEA) has been widely applied to evaluate environmental performance. As a nonparametric approach, it has the advantage that it does not require any prior assumptions on the underlying functional relationships between variables of inputs and outputs. It is based on data-driven frontier analysis, and it floats a piecewise linear surface to rest on top of the observed quantities of inputs and outputs (see [7-11]). And, DEA has gained many new developments as a large amount of papers related to variation of models, and DEA applications have been published. DEA has been widely applied in environmental performance evaluation.

Many scholars have published papers on the evaluation of environmental performance by DEA. The main issue when using DEA models to evaluate the environmental performance is to deal with undesirable outputs [12]. Many methods are proposed in DEA models to deal with undesirable outputs, for example, the data translation approach (e.g., [13-15]), treating undesirable outputs as inputs (e.g., $[1,16-18]$ ), environmental DEA technology (e.g., [19-22]). However, the existing literature deems each DMU as a black box but neglects that some real production systems may have network structure as a two-stage network. 
In the real production process, the internal structure of DMU's production system is often a two-stage production process ([23-29]; Liu et al. 2018). Thus, the two-stage structure of the production system should be considered when evaluating the environmental performance of this kind of production system. In previous literature, Song et al. [29] deemed the Chinese industry system as a twostage production system but treated the two subsystems as two independent subsystems which ignored that the intermediate should be consistent in the two subsystems. Recently, Wu et al. [35] proposed a two-stage DEA model with shared inputs to evaluate the environmental performance of the Chinese industry, without considering whether there exists a cooperative relationship between two subsystems. Lozano [36] computes the environmental performance of a two-stage production and abatement system by a network slacks-based inefficiency measure. Liu et al. [32] further extended two-stage DEA models with undesirable indexes by considering undesirable input-intermediate-outputs.

In reality, the two-stage production system usually has the feature that one subsystem dominates the other, for example, there are usually two participants in a two-stage supply chain, manufacturer and retailer. In such a case, the manufacturer usually holds manipulative power and acts as a leader, and the retailer acts as a follower in non-cooperative supply chains models [35]. Thus, when evaluating the efficiency of such systems, the leader and follower relationship should be considered (e.g., [35, 36]). In the existing literature, some scholars have proposed to model this kind of leader and follower relationship by introducing Stackelberg game theory in the efficiency evaluation. For example, Liang et al. [35] firstly considers the leader-follower relationship in the efficiency evaluation of two-stage supply chain, which deemed the manufacturer and retailer as leader and follower, respectively Zha and Liang [36] considered shared inputs among different stages, which further extended the noncooperative two-stage approach. Li et al. [32] further extended Liang et al.'s [35] non-cooperative two-stage approach by considering additional inputs to stage two. Tavana and Khalili-Damghani [35] further considered the fuzzy data in the two-stage production process by proposing a new two-stage Stackelberg fuzzy DEA model.

The aim of the paper is intended to calculate environmental efficiency for two-stage production systems based on Stackelberg game theory. We use a non-cooperative twostage approach proposed by Liang et al. [35] to measure the environmental efficiencies of the Chinese regional industry system. Especially, the leader-follower relationship between the subsystem efficiencies will be investigated. And, by considering undesirable intermediates and undesirable outputs, we proposed a new non-cooperative two-stage DEA model to measure the environmental efficiency. Finally, the new proposed method is applied to measure the environmental efficiencies of Chinese regional industry systems.

The structure of this paper is organized as follows. In Section 2, we propose a new non-cooperative two-stage DEA model to measure the environmental performance of the two-stage production system. In Section 3, the new proposed approach is applied to Chinese regional industry systems in 2010. Conclusions are drawn in Section 4.

\section{Environmental Efficiency Measurement of Chinese Industry Systems by Using Non-Cooperative Two-Stage DEA Model}

Figure 1 shows a two-stage production process. Suppose there exist $n$ DMUs. Each DMU is denoted as $\operatorname{DMU}_{j}(1,2, \ldots, n)$. In the production subsystem, inputs $x_{i j}^{1}, i=1, \ldots, m$ are used to produce desirable outputs $y_{r j}^{1}, r=1, \ldots, s$ and undesirable outputs $p_{d k}^{1}, d=1, \ldots, D$. Here, $p_{d k}^{1}, d=1, \ldots, D$ are denoted as intermediate measures. In the pollutant treatment subsystem, they are also used as the inputs. The exogenous inputs of the pollutant treatment subsystem are denoted as $x_{h j}^{2}, h=1, \ldots, H$. In the pollutant treatment subsystem, the desirable outputs and undesirable outputs are $y_{g j}^{2}, g=1, \ldots, G$ and $p_{b j}^{2}, b=1, \ldots, B$, respectively.

If the DMU is deemed as a black box, and the internal structure is ignored, then $\mathrm{DMU}_{j}$ 's inputs and outputs are as follows: $x_{i j}^{1}, i=1, \ldots, m$ and $x_{h k}^{2}, h=1, \ldots, H$ are inputs, $y_{r j}^{1}, r=1, \ldots, s$ and $y_{g j}^{2}, g=1, \ldots, G$ are desirable outputs, and $p_{b j}^{2}, b=1, \ldots, B$ are undesirable outputs. As for the undesirable outputs, pollutants are usually the by-products of the desirable outputs. Thus, in the measurement of environmental efficiency, it is expected as little as possible.

To approach the undesirable outputs, a translation method proposed by Seiford and Zhu [36] is adopted to deal with the undesirable outputs. Firstly, each undesirable output is first multiplied by " -1 ." Then, to make the undesirable output positive, we add an appropriate translation vector $v^{2}$ to it. That is, $\overline{p_{b j}^{2}}=-p_{b j}^{2}+v_{b}^{2}, b=1, \ldots, B$, where $v_{b}^{2}$ could be obtained by $v_{b}^{2}=\max _{j}\left\{p_{b j}^{2}\right\}+1, b \in B$. Thus, the larger the $p_{b j}^{2}, b=1, \ldots, B$, the smaller the $\overline{p_{b j}^{2}}, b=1, \ldots, B$. As for evaluating the environmental efficiency of $\mathrm{DMU}_{k}$ when deeming it as a black box, we can apply the following CCR model (see [37] to obtain it):

$$
\begin{aligned}
& e_{k}^{\mathrm{CCR}}= \max \frac{\sum_{r=1}^{s} u_{r} y_{r k}^{1}+\sum_{g=1}^{G} \pi_{g} y_{g k}^{2}+\sum_{b=1}^{B} \varepsilon_{b} \overline{p_{b k}^{2}}}{\sum_{i=1}^{m} v_{i} x_{i k}^{1}+\sum_{h=1}^{H} Q_{h} x_{h k}^{2}}, \\
& \text { s.t. } \frac{\sum_{r=1}^{s} u_{r} y_{r j}^{1}+\sum_{g=1}^{G} \pi_{g} y_{g j}^{2}+\sum_{b=1}^{B} \varepsilon_{b} \overline{p_{b j}^{2}}}{\sum_{i=1}^{m} v_{i} x_{i j}^{1}+\sum_{h=1}^{H} Q_{h} x_{h j}^{2}} \leq 1, \quad \forall j, \\
& u_{r}, v_{i}, \pi_{g}, \varepsilon_{b}, Q_{h} \geq 0, \quad \forall i, r, g, b, h .
\end{aligned}
$$

In the production subsystem, the nonnegative weights $v_{i}$ and $u_{r}$ are attached to its inputs and desirable outputs, respectively. And, in the pollutant treatment subsystem, the nonnegative weights $Q_{h}, \pi_{g}$, and $\varepsilon_{b}$ are attached to inputs, desirable outputs, and undesirable outputs, respectively. $e_{k}^{\mathrm{CCR} *}$ is the optimal value of model (1), which is the environmental efficiency of $\mathrm{DMU}_{k}$ when $\mathrm{DMU}_{k}$ is deemed as a black box. Based on this approach, each DMU's internal structure is ignored. Thus, we could not identify the environmental efficiencies of both subsystems. 


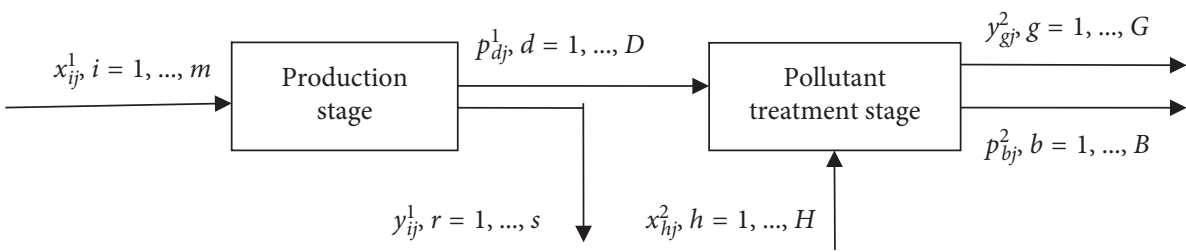

FIGURE 1: The two-stage production system.

The study aims to measure the environmental efficiencies of DMUs with a production structure of two-stage network. In this paper, the non-cooperative two-stage DEA approach proposed by Liang et al. [35] is adopted in this study. When the first subsystem is assumed to be more important, it is deemed as a leader, and the second subsystem is deemed as a follower. For a specific $\mathrm{DMU}_{k}$, the environmental efficiency of its first subsystem (the leader) could be calculated by the following traditional CCR model [37]:

$$
\begin{aligned}
E_{k}^{1 *}= & \operatorname{Max} \frac{\sum_{d=1}^{D} w_{d} \bar{z}_{d k}+\sum_{r_{P}=1}^{s_{P}} H_{r_{P}} y_{r_{P} k}}{\sum_{i_{P}=1}^{m_{P}} v_{i_{P}} x_{i_{P} k}}, \\
& \text { s.t. } \frac{\sum_{d=1}^{D} w_{d} \bar{z}_{d j}+\sum_{r_{p}=1}^{s_{P}} H_{r_{p}} y_{r_{p} j}}{\sum_{i_{P}=1}^{m_{P}} v_{i_{P}} x_{i_{P} j}} \leq 1, \quad \forall j, \\
& v_{i_{P}}, w_{d}, H_{r_{P}} \geq 0, \quad \forall i_{P}, \forall d, \forall H_{r_{P}}, \\
& u_{r}, v_{i}, \pi_{g}, \varepsilon_{b}, Q_{h} \geq 0, \quad \forall i, r, g, b, h .
\end{aligned}
$$

In the production subsystem, the nonnegative weights $v_{i_{p}}$, $w_{d}$, and $H_{r_{p}}$ are attached to inputs, intermediates, and desirable outputs, respectively. The optimal environmental efficiency of this subsystem is $E_{k}^{1 *}$, which is the optimal value of model (2). In model (2), the undesirable outputs are $z_{d j}, d=1, \ldots, D$. To deal with the undesirable outputs, we firstly multiply " -1 " by each undesirable output and then add an appropriate translation vector $p$ to the negative undesirable outputs. Then, the undesirable outputs are positive by this approach. That is, $\overline{z_{d j}}=-z_{d j}+p_{d}, d=1, \ldots, D$, and $p_{d}$ is obtained by $p_{d}=\max _{j}\left\{z_{d j}\right\}+1, d \in D$. In this approach, the larger the $z_{d j}, d=1, \ldots, D$, the smaller the $\overline{z_{d j}}, d=1, \ldots, D$.

Model (2) is a fractional program, but we can apply Charnes-Cooper (C-C) transformation to convert it into a linear model as follows:

$$
\begin{aligned}
E_{k}^{1 *}= & \operatorname{Max} \sum_{d=1}^{D} w_{d} \bar{z}_{d k}+\sum_{r_{P}=1}^{s_{P}} H_{r_{P}} y_{r_{p} k}, \\
& \text { s.t. } \sum_{d=1}^{D} w_{d} \bar{z}_{d j}+\sum_{r_{p}=1}^{s_{P}} H_{r_{p}} y_{r_{p} j}-\sum_{i_{P}=1}^{m_{P}} v_{i_{P}} x_{i_{P} j} \leq 0, \quad \forall j, \\
& \sum_{i_{P}=1}^{m_{P}} v_{i_{P}} x_{i_{P} k}=1, \\
& v_{i_{P}}, w_{d}, H_{r_{P}} \geq 0, \quad \forall i_{P}, \forall d, \forall H_{r_{P}} .
\end{aligned}
$$

The optimal environmental efficiency of the production subsystem is obtained by model (3). The intermediate measure connects the two subsystems. When the environmental efficiency of the production subsystem is obtained, the pollutant treatment subsystem will consider the variables $v_{i_{p}}, w_{d}$, and $H_{r_{p}}$ which maintain $E_{k}^{1}=E_{k}^{1 *}$. Following the non-cooperative two-stage concept of Liang et al. [35] and Zha and Liang [36]; we propose the following model that maximizes the environmental efficiency of pollutant treatment subsystem in the objective function with the constraint that maintains $E_{k}^{1}=E_{k}^{1 *}$.

$$
\begin{aligned}
E_{k}^{2 *}= & \operatorname{Max} \frac{\sum_{b=1}^{B} \varepsilon_{b} \bar{u}_{b k}+\sum_{r_{T}=1}^{s_{T}} R_{r_{T}} y_{r_{T} k}}{\sum_{d=1}^{D} w_{d} \bar{z}_{d k}+\sum_{i_{T}=1}^{m_{T}} \pi_{i_{T}} x_{i_{T} k}}, \\
& \text { s.t. } \frac{\sum_{d=1}^{D} w_{d} \bar{z}_{d j}+\sum_{r_{P}=1}^{P} H_{r_{P}} y_{r_{P} j}}{\sum_{i_{P}=1}^{m_{P}} v_{i_{P}} x_{i_{P} j}} \leq 1, \quad \forall j, \\
& \frac{\sum_{b=1}^{B} \varepsilon_{b} \bar{u}_{b j}+\sum_{r_{T}=1}^{s_{T}} R_{r_{T}} y_{r_{T} j}}{\sum_{d=1}^{D} w_{d} \bar{z}_{d j}+\sum_{i_{T}=1}^{m_{T}} \pi_{i_{T}} x_{i_{T} j}} \leq 1, \quad \forall j, \\
& \frac{\sum_{d=1}^{D} w_{d} \bar{z}_{d k}+\sum_{r_{P}=1}^{s_{P}} H_{r_{P}} y_{r_{P}} k}{\sum_{i_{P}=1}^{m_{P}} v_{i_{P}} x_{i_{P} k}}=E_{k}^{1 *} .
\end{aligned}
$$

In model (4), the nonnegative weights $\pi_{i_{T}}, R_{r_{T}}$, and $\varepsilon_{b}$ are attached to inputs, desirable outputs, and undesirable outputs in the pollutant treatment subsystem, respectively. The optimal environmental efficiency of this subsystem is $E_{k}^{2 *}$, which is the optimal value of model (4). In model (4), the undesirable outputs are $u_{b j}, b=1, \ldots, B$. To deal with the undesirable outputs, we firstly multiply “ -1 ” by each undesirable output and then add an appropriate translation vector $q$ to the negative undesirable outputs. Then, the undesirable outputs are positive by this approach. That is, $\overline{u_{b k}}=-u_{b k}+q_{b},(b=1, \ldots, B)$, and $q_{b}$ is obtained by $q_{b}=\max \left\{u_{b k}\right\}+1, b \in B$. In this approach, the larger the $u_{b k}, b=1, \ldots, B$, the smaller the $\overline{u_{b k}}$. In model (4), the environmental efficiency of $\mathrm{DMU}_{k}^{\prime}$ pollutant treatment subsystem is maximized with the constraint that the environmental efficiency of the production subsystem remains unchanged. Model (4) is a fractional program, which could be transformed to be a linear program as follows: 


$$
\begin{aligned}
E_{k}^{2 *}= & \operatorname{Max} \sum_{b=1}^{B} \varepsilon_{b} \bar{u}_{b k}+\sum_{r_{T}=1}^{s_{T}} R_{r_{T}} y_{r_{T} k}, \\
& \text { s.t. } \sum_{d=1}^{D} w_{d} \bar{z}_{d k}+\sum_{i_{T}=1}^{m_{T}} \pi_{i_{T}} x_{i_{T} k}=1, \\
& \sum_{d=1}^{D} w_{d} \bar{z}_{d j}+\sum_{r_{P}=1}^{s_{P}} H_{r_{P}} y_{r_{P} j}-\sum_{i_{P}=1}^{m_{P}} v_{i_{P}} x_{i_{P} j} \leq 0, \quad \forall j, \\
& \sum_{b=1}^{B} \varepsilon_{b} \bar{u}_{b j}+\sum_{r_{T}=1}^{s_{T}} R_{r_{T}} y_{r_{T} j}-\sum_{d=1}^{D} w_{d} \bar{z}_{d j}-\sum_{i_{T}=1}^{m_{T}} \pi_{i_{T}} x_{i_{T} j} \leq 0, \quad \forall j, \\
& \sum_{d=1}^{D} w_{d} \bar{z}_{d k}+\sum_{r_{P}=1}^{s_{P}} H_{r_{P}} y_{r_{P} k}=E_{k}^{1 *} \sum_{i_{P}=1}^{m_{P}} v_{i_{P}} x_{i_{P} k} .
\end{aligned}
$$

The optimal value of model (5) is denoted as $E_{k}^{2 *}$, which is the optimal environmental efficiency of the pollutant treatment subsystem. Then, for the overall system, $\mathrm{DMU}_{k}^{\prime}$ environmental efficiency is $E^{\text {non, } 1}=E_{k}^{1 *} * E_{k}^{2 *}$.

In a similar approach, if the pollutant treatment subsystem is assumed to be the leader, $\mathrm{DMU}_{k}^{\prime}$ environmental efficiency of the pollutant treatment subsystem $\pi_{k}^{2 *}$ could be calculated by traditional CCR model with inputs $\left(z_{d k}\right.$ and $\left.x_{i_{p} k}\right)$ and desirable outputs $\left(y_{r_{p} k}\right)$, and undesirable outputs $\left(u_{b k}\right)$. Then, we could calculate the environmental efficiency for the production subsystem $\pi_{k}^{1 *}$ by solving a DEA model that maintains $\pi_{k}^{2}=\pi_{k}^{2 *}$. Then, $\mathrm{DMU}_{k}^{\prime}$ efficiency for the overall system is $E^{n o n, 2}=\pi_{k}^{1 *} * \pi_{k}^{2 *}$.

\section{Application to Regional Industry Systems in China}

In previous literature of evaluating the environmental efficiency, most studies treat the production process of Chinese regional industry systems as a black box and neglect its network structure. To overcome the problem, we consider the internal structure of Chinese regional industry systems by dividing the process into two subsystems: production subsystem and pollutant treatment subsystem. As the status of these two subsystems may not be equal, we consider the non-cooperative relationship between these two subsystems. In this section, the proposed non-cooperative two-stage DEA model will be applied to measure the environmental efficiency of Chinese regional industry systems. And, we provide some practical suggestions for governments to govern environmental protection in industrial sectors.

In this section, the environmental efficiency of Chinese regional industry systems in the year 2010 will be analyzed by applying our non-cooperative two-stage DEA approach. We do not use the data after 2010 as the indicators of the statistical system and statistical technologies were revised by the Ministry of Environmental Protection after 2010 (some indicators are absent). As shown in Figure 1, each regional industry system is divided into two subsystems: production subsystem and pollutant treatment subsystem.
As for selecting the variables, we follow the existing work of Song et al. [29]. In the production subsystem, inputs- resources are utilized to generate desirable outputproducts and undesirable outputs-pollutants. The pollutants from the production subsystem are then disposed in the pollutant treatment subsystem by using pollutant investments. In the pollutant treatment subsystem, the desirable output is the value of comprehensive utilization of the three wastes (VCU), and the undesirable outputs are the emitted pollutants. In the production subsystem, we select the total amount of employees, fixed assets, and electricity as inputs. We select gross domestic product (GDP) as the desirable output as it can reflect the production subsystem intuitively. We select $\mathrm{COD}, \mathrm{SO}_{2}$, and solid waste generated from the production subsystem as undesirable outputs as they are harmful. And, they are selected as re-inputs as they are disposed in the pollutant treatment subsystem. In the pollutant treatment subsystem, we select the value of comprehensive utilization of the three wastes (VCU) as desirable outputs, and COD, $\mathrm{SO}_{2}$, and solid waste generated as undesirable outputs. We obtain the data from China Statistical Yearbook 2010 published by the China National Bureau of Statistics in 2010. Some statistical data of Tibet are not available, thus Tibet is excluded in our study. The characteristics of the data are documented in Table 1.

In Table 2, we list the results of overall efficiency, production efficiency, and pollutant treatment efficiency based on our proposed approach. In order to analyze the difference that existed among the regions in terms of the overall efficiency scores when the production subsystem is a leader and the pollutant treatment subsystem is a leader, we present the cluster analysis map of the overall efficiency level of Chinese 30 regional industry systems in Figures 2 and 3, respectively.

According to Figure 2, when the production subsystem is a leader, the overall efficiency scores of 30 regional industry systems are significantly different. In the eastern area, 5 out of 11 regions (Beijing (P1), Tianjin (P2), Shanghai (P5), Zhejiang (P7), and Hainan (P11)) are highly efficient as their overall efficiency scores are above $0.8,1$ out of 11 regions (Guangdong (P10)) has overall efficiency scores between 0.6 and $0.8,1$ out of 11 regions (Fujian (P8)) has overall efficiency scores between 0.4 and 0.6 , and 3 out of 11 regions (Hebei (P3), Liaoning (P4), Jiangsu (P6), and Shandong (P9)) are worst with overall efficiency scores below 0.3 . In the central area, 2 out of 10 regions (Inner Mongolia (P13) and Jilin (P14)) obtain the highest overall efficiency scores, 1 out of 10 regions (Heilongjiang (P15)) has overall efficiency scores between 0.6 and 0.8 , and 1 out of 10 regions (Guangxi (P21)) has an overall efficiency score between 0.4 and 0.6. Shanxi (P12), Anhui (P16), Jiangxi (P17), Henan (P18), Hubei (P19), and Hunan (P20) are the six worst DMUs with overall efficiency scores below 0.4. In the western area, Qinghai (P28) obtains the highest overall efficiency scores as it has an overall efficiency score above $0.8,3$ out of 9 regions (Chongqing (P22), Ningxia (P29), and Xinjiang (P30)) have overall efficiency scores between 0.6 and $0.8,2$ out of 9 regions (Yunnan (P25) and Shaanxi (P26)) have overall 
Table 1: Descriptive statistics of raw data.

\begin{tabular}{|c|c|c|c|c|c|}
\hline Variables & Units & Max & Min & Mean & Standard deviation \\
\hline Employees & 10000 persons & 2141.907 & 53.568 & 734.312 & 636.557 \\
\hline Fixed assets & 1 billion yuan & 12372.821 & 177.744 & 3810.847 & 2940.517 \\
\hline Electricity consumption & $(100$ million $\mathrm{kW} \cdot \mathrm{h})$ & 4060.130 & 159.020 & 1399.280 & 1012.942 \\
\hline COD generation & 10000 tons & 261.256 & 4.237 & 61.668 & 59.218 \\
\hline $\mathrm{SO}_{2}$ generation & 10000 tons & 453.535 & 11.598 & 172.277 & 106.494 \\
\hline Solid waste generation & 10000 tons & 31688.000 & 212.000 & 8031.000 & 6635.777 \\
\hline GDP of the second industry & 1 billion yuan & 21462.720 & 385.210 & 6442.604 & 5475.785 \\
\hline Pollutant investment & 1 billion yuan & 456759.300 & 4353.500 & 132325.607 & 105065.660 \\
\hline $\mathrm{VCU}$ & 10000 yuan & 49.268 & 0.488 & 14.488 & 10.878 \\
\hline COD emission & 10000 tons & 138.287 & 2.817 & 62.144 & 36.129 \\
\hline $\mathrm{SO}_{2}$ emission & 10000 tons & 12011.451 & 0.001 & 1925.202 & 2644.794 \\
\hline Solid waste emission & 10000 tons & 2863867.000 & 31623.000 & 592826.533 & 650121.434 \\
\hline
\end{tabular}

TABLE 2: Environmental efficiency scores of all regions.

\begin{tabular}{|c|c|c|c|c|c|c|c|c|}
\hline \multirow[b]{2}{*}{ DMU } & \multirow[b]{2}{*}{ Region } & \multicolumn{3}{|c|}{ Subsystem 1 as a leader } & \multicolumn{3}{|c|}{ Subsystem 2 as a leader } & \multirow{2}{*}{$\begin{array}{c}\text { Black-box } \\
\text { CCR }\end{array}$} \\
\hline & & $E^{\text {non }, 1}$ & $E_{k}^{1 *}$ & $E_{k}^{2 *}$ & $E^{\text {non,2 }}$ & $\pi_{k}^{1 *}$ & $\pi_{k}^{2 *}$ & \\
\hline \multicolumn{9}{|c|}{ Eastern area } \\
\hline 1 & Beijing & 0.9949 & 1.0000 & 0.9949 & 0.9949 & 1.0000 & 0.9949 & 0.3566 \\
\hline 2 & Tianjin & 0.9368 & 1.0000 & 0.9368 & 0.9368 & 1.0000 & 0.9368 & 0.6395 \\
\hline 3 & Hebei & 0.2557 & 0.6047 & 0.4228 & 0.6047 & 0.6047 & 1.0000 & 0.5653 \\
\hline 4 & Liaoning & 0.0787 & 0.7558 & 0.1041 & 0.5668 & 0.7558 & 0.7499 & 0.3431 \\
\hline 5 & Shanghai & 0.9769 & 1.0000 & 0.9769 & 0.9769 & 1.0000 & 0.9769 & 0.3756 \\
\hline 6 & Jiangsu & 0.3954 & 0.7981 & 0.4954 & 0.7981 & 0.7981 & 1.0000 & 0.6724 \\
\hline 7 & Zhejiang & 0.8115 & 0.8115 & 1.0000 & 0.8115 & 0.8115 & 1.0000 & 1.0000 \\
\hline 8 & Fujian & 0.5358 & 0.8517 & 0.6290 & 0.8479 & 0.8479 & 1.0000 & 0.3574 \\
\hline 9 & Shandong & 0.1582 & 0.9132 & 0.1732 & 0.9132 & 0.9132 & 1.0000 & 0.6540 \\
\hline 10 & Guangdong & 0.7690 & 1.0000 & 0.7690 & 0.7690 & 1.0000 & 0.7690 & 0.2271 \\
\hline \multirow{2}{*}{11} & Hainan & 1.0000 & 1.0000 & 1.0000 & 1.0000 & 1.0000 & 1.0000 & 1.0000 \\
\hline & Average & 0.6284 & 0.8850 & 0.6820 & 0.8382 & 0.8847 & 0.9480 & 0.5628 \\
\hline \multicolumn{9}{|c|}{ Central area } \\
\hline 12 & Shanxi & 0.3314 & 0.5955 & 0.5564 & 0.5651 & 0.5810 & 0.9725 & 0.5649 \\
\hline 13 & Inner Mongolia & 1.0000 & 1.0000 & 1.0000 & 1.0000 & 1.0000 & 1.0000 & 0.7123 \\
\hline 14 & Jilin & 0.9602 & 1.0000 & 0.9602 & 0.9658 & 0.9989 & 0.9669 & 1.0000 \\
\hline 15 & Heilongjiang & 0.7600 & 0.9522 & 0.7981 & 0.9301 & 0.9396 & 0.9899 & 0.6739 \\
\hline 16 & Anhui & 0.4378 & 0.7967 & 0.5495 & 0.7344 & 0.7344 & 1.0000 & 0.6055 \\
\hline 17 & Jiangxi & 0.5053 & 0.9413 & 0.5368 & 0.8959 & 0.8959 & 1.0000 & 0.9578 \\
\hline 18 & Henan & 0.2086 & 0.7935 & 0.2629 & 0.7662 & 0.7905 & 0.9692 & 0.3441 \\
\hline 19 & Hubei & 0.1943 & 0.8173 & 0.2378 & 0.7382 & 0.8157 & 0.9050 & 0.6699 \\
\hline 20 & Hunan & 0.2438 & 0.8489 & 0.2872 & 0.7334 & 0.8380 & 0.8751 & 0.8293 \\
\hline \multirow[t]{2}{*}{21} & Guangxi & 0.5955 & 0.7289 & 0.8169 & 0.6168 & 0.6630 & 0.9304 & 0.5963 \\
\hline & Average & 0.5237 & 0.8474 & 0.6006 & 0.7946 & 0.8257 & 0.9609 & 0.6954 \\
\hline \multicolumn{9}{|c|}{ Western area } \\
\hline 22 & Chongqing & 0.7845 & 0.9489 & 0.8267 & 0.8346 & 0.9389 & 0.8889 & 0.6388 \\
\hline 23 & Sichuan & 0.2240 & 0.7736 & 0.2895 & 0.6235 & 0.7736 & 0.8059 & 0.3353 \\
\hline 24 & Guizhou & 0.3837 & 0.4294 & 0.8935 & 0.4212 & 0.4212 & 1.0000 & 0.4755 \\
\hline 25 & Yunnan & 0.4184 & 0.5153 & 0.8121 & 0.5054 & 0.5054 & 1.0000 & 1.0000 \\
\hline 26 & Shaanxi & 0.3557 & 0.8670 & 0.4102 & 0.6584 & 0.8639 & 0.7622 & 0.4484 \\
\hline 27 & Gansu & 0.2474 & 0.4123 & 0.6001 & 0.4462 & 0.4462 & 1.0000 & 0.6937 \\
\hline 28 & Qinghai & 0.8805 & 0.9227 & 0.9542 & 0.9061 & 0.9061 & 1.0000 & 0.9728 \\
\hline 29 & Ningxia & 0.6207 & 0.6872 & 0.9031 & 0.6381 & 0.6799 & 0.9384 & 0.9091 \\
\hline \multirow[t]{3}{*}{30} & Xinjiang & 0.6995 & 0.9217 & 0.7589 & 0.8244 & 0.9110 & 0.9050 & 1.0000 \\
\hline & Average & 0.5127 & 0.7198 & 0.7165 & 0.6509 & 0.7163 & 0.9223 & 0.7193 \\
\hline & National average & 0.5588 & 0.8229 & 0.6652 & 0.7675 & 0.8145 & 0.9446 & 0.6540 \\
\hline
\end{tabular}

efficiency scores between 0.4 and 0.6 , and 3 out of 9 regions (Sichuan (P23), Guizhou (P24), and Gansu (P27)) have overall efficiency scores below 0.4 .
According to Figure 3, when the pollutant treatment subsystem is a leader, the overall efficiency scores of 30 regional industry systems are also significantly different. In 


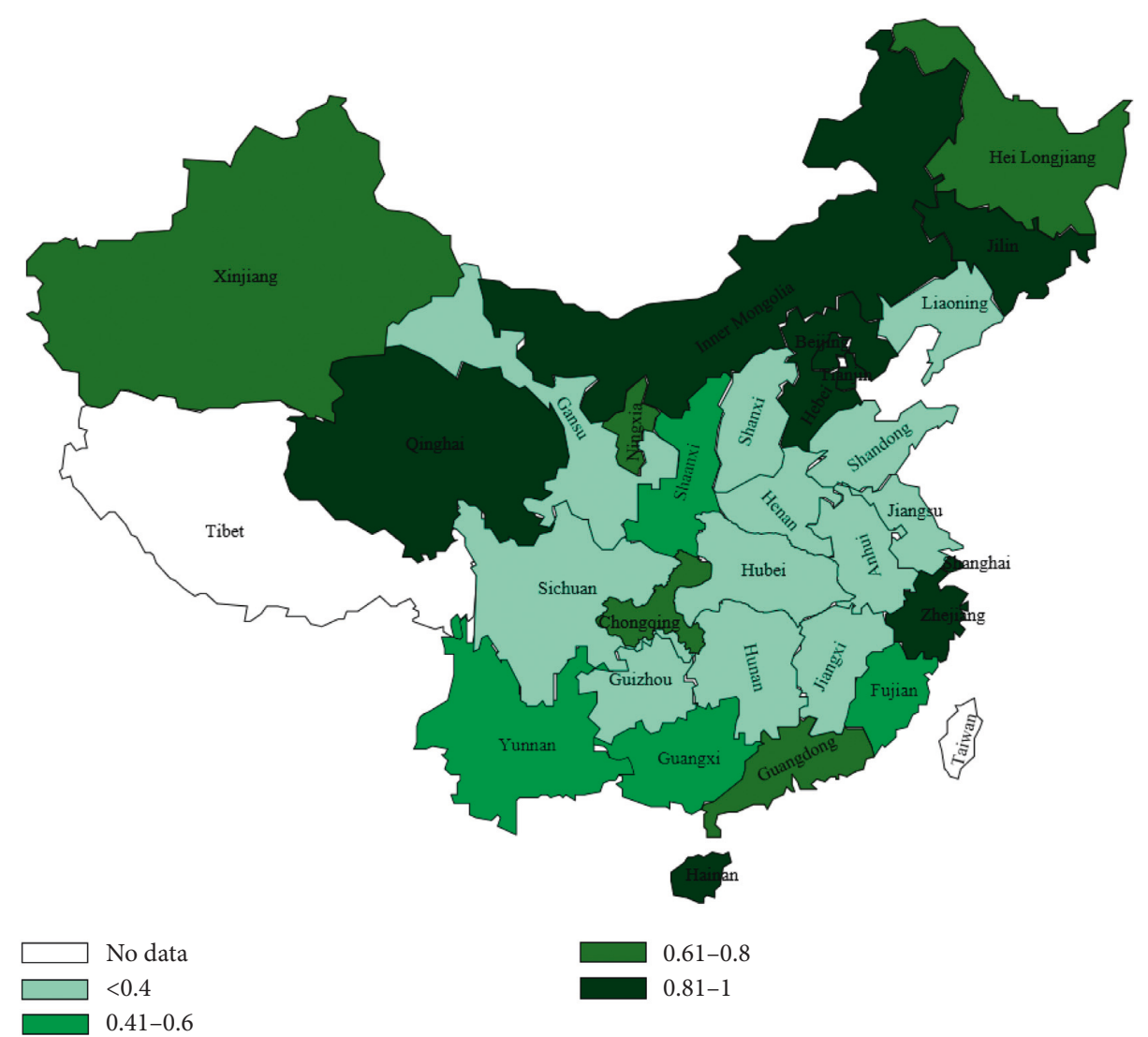

FIGURE 2: Cluster analysis map of the overall efficiency level of 30 Chinese regional industry systems when subsystem 1 is a leader.

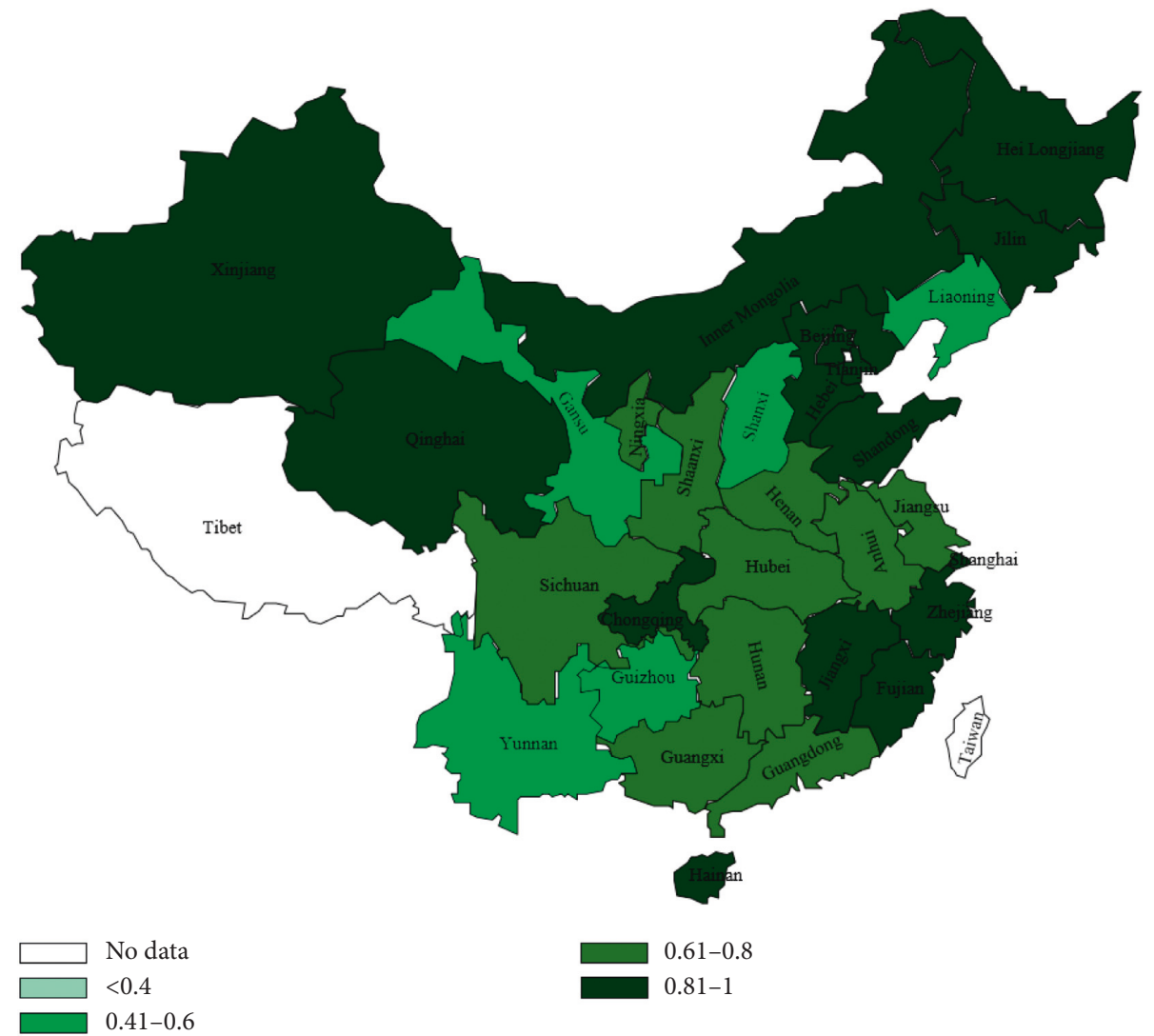

FIGURE 3: Cluster analysis map of overall efficiency level of 30 Chinese regional industry systems when subsystem 2 is a leader. 
the eastern area, 7 out of 11 regions (Beijing (P1), Tianjin (P2), Shanghai (P5), Zhejiang (P7), Fujian (P8), and Shandong (P9)) are highly efficient as their overall efficiency scores are above 0.8 , and 3 out of 11 regions (Hebei (P3), Jiangsu (P6), and Guangdong (P10)) have overall efficiency scores between 0.6 and 0.8 . In the central area, 6 out of 10 regions (Inner Mongolia (P13), Jilin (P14), Heilongjiang (P15), and Jiangxi (P17)) obtain the highest overall efficiency scores, 5 out of 10 regions (Anhui (P16), Henan (P18), Hubei (P19), Hunan (P20), and Guangxi (P21)) have overall efficiency scores between 0.6 and 0.8 . Shanxi (P12) is the worst $\mathrm{DMU}$ with overall efficiency scores below 0.6 . In the western area, 3 out of 9 regions (Chongqing (P22), Qinghai (P28), and Xinjiang (P30)) are highly efficient as their overall efficiency scores are above $0.8,3$ out of 9 regions (Sichuan (P23), Shaanxi (P26), and Ningxia (P29)) have overall efficiency scores between 0.6 and 0.8. Guizhou (P24), Yunnan (P25), and Gansu (P27) are the three worst DMUs with overall efficiency scores below 0.6 .

The results of the environmental efficiencies when deeming the DMUs as "black box" and the environmental efficiencies based on our new proposed approach are documented in Table 2. When the production subsystem is assumed to be a leader, the corresponding maximum environmental efficiency of this subsystem $E_{k}^{1 *}$, the minimum environmental efficiency of the pollutant treatment subsystem $E_{k}^{2 *}$, and the environmental efficiency of the whole system $E^{\text {non,1 }}$ are documented in column 3 to 5 . When the pollutant treatment subsystem is assumed to be a leader, the corresponding maximum environmental efficiency of the production subsystem $\pi_{k}^{1 *}$, the minimum environmental efficiency of the pollutant treatment subsystem $\pi_{k}^{2 *}$, and the environmental efficiency of the whole system $E^{\text {non, } 2}$ are documented in column 6 to 8 . When the internal structure of regional industry system is neglected, the corresponding environmental efficiency $e_{k}^{\mathrm{CCR} *}$ is documented in column 9. It could be found that 5 regions are efficient by using Black-box DEA, while only 2 regions are efficient in the whole system when the production subsystem is considered as a leader in the two-stage production process (it is similar when the pollutant treatment subsystem is considered as a leader). It can be seen that the proposed non-cooperative two-stage DEA model identifies more sources of inefficiency than the traditional Black-Box DEA model. The reason is that the discriminating power of it is better than that of the traditional Black-Box DEA model. Based on our proposed approach, Hainan (P10) and Inner Mongolia (P13) are efficient in the overall system. It could be seen that any DMU is efficient in the overall system if and only if it is efficient in both subsystems.

In order to compare the difference of the environmental efficiencies in three areas (i.e., eastern area, central area, and western area), we compare the efficiency results in Table 2. It could be found that the average environmental efficiency for the overall system in the eastern area at 0.6248 is higher than that in the central area at 0.5237 and western area at 0.5127 . As for the average environmental efficiency for the production subsystem and pollutant treatment subsystem, it could be found that there exists significant efficiency difference between these two subsystems.
Based on the results, suggestions for improving the environmental efficiencies of 30 regional industry systems can be given. In the case that the production subsystem is assumed as a leader and in the case that the pollutant treatment subsystem is assumed as a leader, the environmental efficiency scores of both subsystems are used to plot the efficiency matrix as shown in Figures 4 and 5, respectively. In these two figures, the horizontal axis of the efficiency matrix represents the environmental efficiency for the production subsystem and the vertical axis represents the environmental efficiency for the pollutant treatment subsystem. Each region is located in the matrix. When the production subsystem is a leader, the average environmental efficiency for the production subsystem of the 30 regions is 0.8229 , and that of the pollutant treatment subsystem is 0.6652 ; when the pollutant treatment subsystem is a leader, the average environmental efficiency for the production subsystem of the 30 regions is 0.8145 , and that of the pollutant treatment subsystem is 0.9446 . Thus, the efficiency matrix could be divided into four quadrants using both average environmental efficiencies as shown in Figures 4 and 5. In the case of the production subsystem acting as a leader and that of the pollutant treatment subsystem acting as a leader, policy and strategic suggestions for improving environmental efficiency could be given based on the location of the regions in the four quadrants in Figures 4 and 5, respectively. For example, as seen in Figure 4, when the production subsystem is a leader, Jiangxi (17) is located in the fourth quadrant; it means Jiangxi (17) has large environmental efficiency in the production subsystem while it has small environmental efficiency in the pollutant treatment subsystem. Thus, if Jiangxi (17) wants to improve its environmental efficiency in the overall system, then it should put more emphasis on the pollutant treatment subsystem.

In order to study the impact of pollutant intensities on the overall efficiency of a DMU (e.g., a region or a country), carbon intensity was used to monitor a DMU's environmental performance in previous studies on environmental performance analysis [38]. The previous approach is adopted to define the intensities in both subsystems as follows: Electricity intensity (EI) is defined as the ratio of electricity consumption and GDP, COD-generation intensity (CGI) is defined as the ratio of $\mathrm{COD}$ generation and $\mathrm{GDP}, \mathrm{SO}_{2}$-generation intensity (SGI) is defined as the ratio of $\mathrm{SO}_{2}$ generation and GDP, solid waste-generation intensity (SWGI) is defined as the ratio of solid waste generation and GDP, COD-discharge intensity (CDI) is defined as the ratio of COD discharge and VCU, $\mathrm{SO}_{2}$-discharge intensity (SDI) is defined as the ratio of $\mathrm{SO}_{2}$ discharge and VCU, and solid waste-discharge intensity (SWDI) is defined as the ratio of solid waste discharge and VCU. To describe the relationship between the overall efficiency score and its determinant factors, we formulate the following multiple regression equation:

$$
\begin{aligned}
\mathrm{OE}= & \beta_{0}+\beta_{1} \mathrm{CGI}+\beta_{2} \mathrm{SGI}+\beta_{3} \mathrm{SWGI}+\beta_{4} \mathrm{EI}+\beta_{5} \mathrm{CDI} \\
& +\beta_{6} \mathrm{SDI}+\beta_{7} \mathrm{SWDI}+\varepsilon,
\end{aligned}
$$




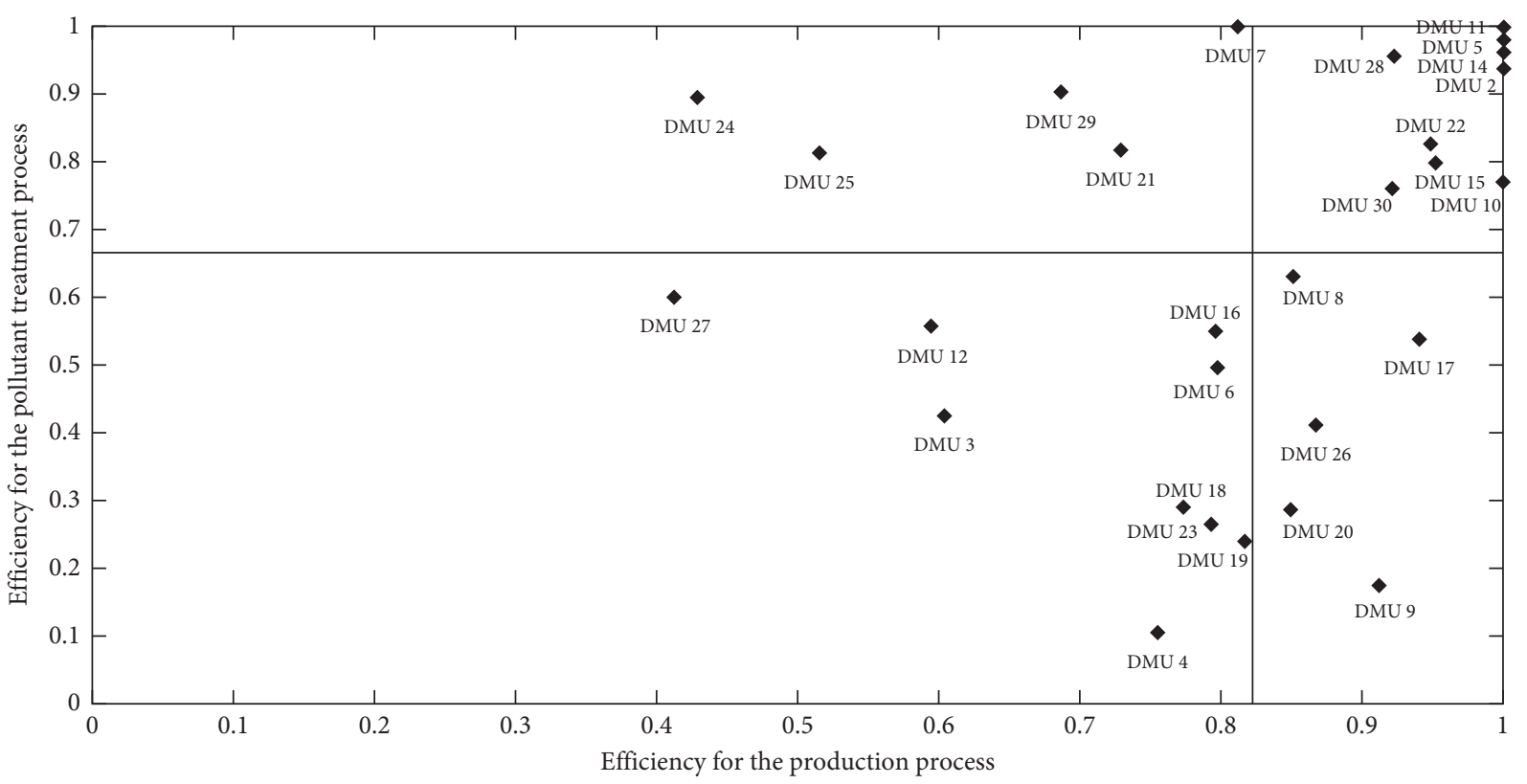

FIGURE 4: Scatter plot of efficiency for two subsystems when the production subsystem is assumed to be a leader.

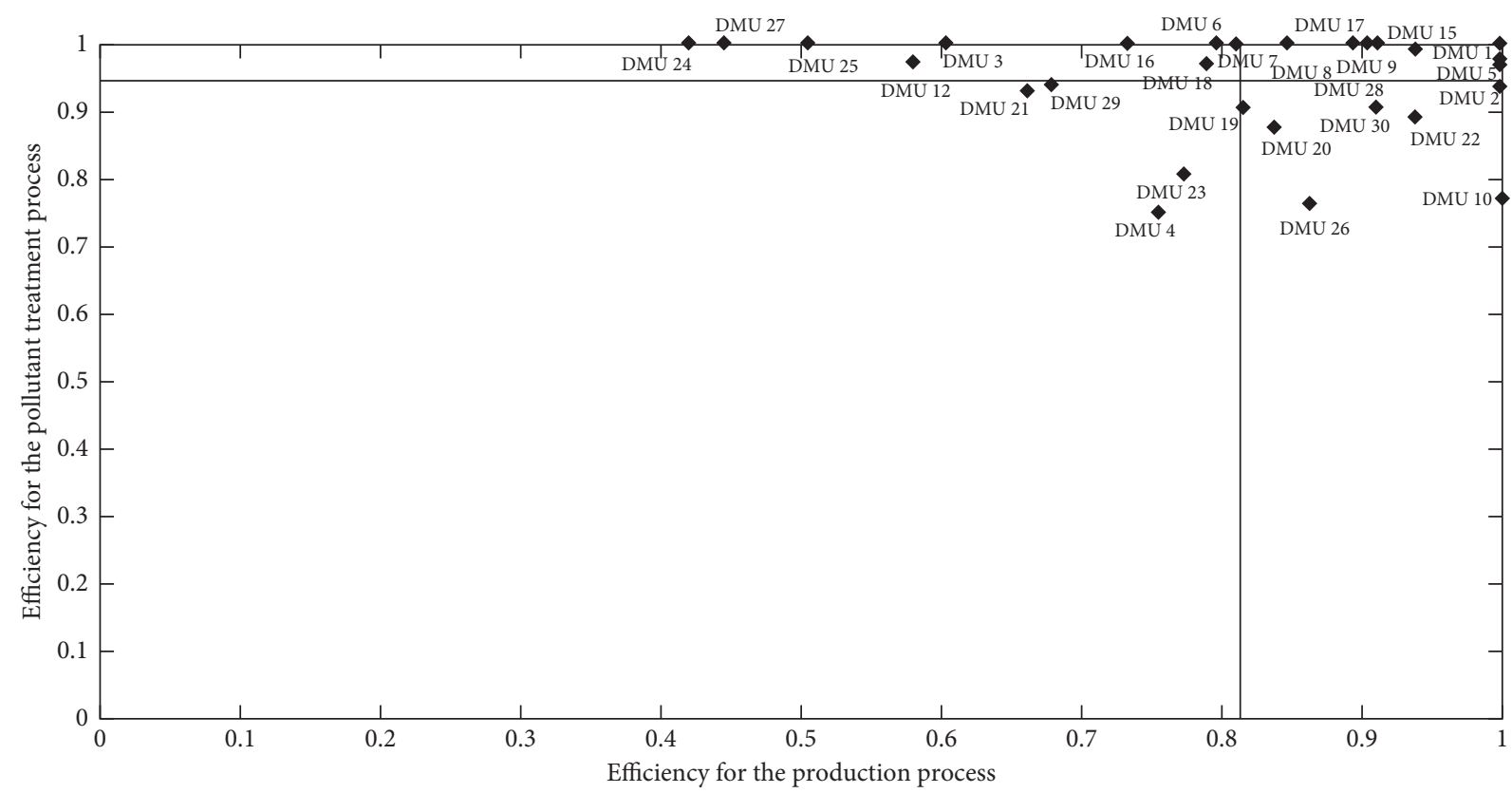

Figure 5: Scatter plot of efficiency for two subsystems when the pollutant treatment subsystem is assumed to be a leader.

where $\varepsilon$ is a random disturbance term such that $\varepsilon \sim N\left(0, o_{\varepsilon}^{2}\right)$.

Tables 3 and 4 summarize the main regression results when the production subsystem is a leader and when the pollutant treatment subsystem is a leader, respectively. The coefficient of determination is denoted as $R^{2}$ or $R^{2}$ (adj). It shows that the multiple regression model fits the data well.

From Tables 3 and 4, we can see that there are three statistically significant determinant factors when the production subsystem is assumed to be a leader, and there are two statistically significant determinant factors when the pollutant treatment subsystem is assumed to be a leader. When the production subsystem is a leader, SGI and SDI have significantly negative relationships with the overall efficiency, EI has significantly positive relationship with the overall efficiency, while CGI, SWGI, CDI, and SWDI have no significant relationship with the overall efficiency. Thus, for the government, to improve the overall environmental performance, more efforts should be made to reduce the emission of $\mathrm{SO}_{2}$ in the production subsystem and $\mathrm{SO}_{2}$ in the pollutant treatment subsystem and make full use of the electricity in the production subsystem. If the pollutant treatment subsystem is assumed to be a leader, two determinant factors (SGI and SDI) have significantly negative relationships with the overall efficiency, while CGI, SWGI, 
TABle 3: Determinants of the overall efficiency scores when subsystem 1 is a leader.

\begin{tabular}{lcc}
\hline Independent variables & Coefficients & Standard error \\
\hline Constant & $0.211^{* * *}$ & 0.156 \\
CGI & 0.148 & 0.259 \\
SGI & $-0.591^{* *}$ & 0.323 \\
SWGI & -0.430 & 0.302 \\
EI & $0.474^{* *}$ & 0.257 \\
CDI & -0.280 & 0.292 \\
SDI & $-0.835^{* *}$ & 0.307 \\
SWDI & -0.192 & 0.232 \\
\hline
\end{tabular}

Note: $R^{2}=0.667$ and $R^{2}$ (adj) $=0.603$. Dependent variables are the overall efficiency scores of each province. ${ }^{* * *},{ }^{* *}$, and ${ }^{*}$ indicate the parameter estimate is significantly different from zero at $0.001,0.01$, and 0.05 , respectively.

TABLE 4: Determinants of the overall efficiency scores when subsystem 2 is a leader.

\begin{tabular}{lcc}
\hline Independent variables & Coefficients & Standard error \\
\hline Constant & $0.137^{* *}$ & 0.109 \\
CGI & 0.259 & 0.181 \\
SGI & $-0.990^{* * *}$ & 0.226 \\
SWGI & -0.351 & 0.211 \\
EI & 0.294 & 0.180 \\
CDI & -0.413 & 0.205 \\
SDI & $-0.633^{* *}$ & 0.215 \\
SWDI & -0.111 & 0.162 \\
\hline
\end{tabular}

Note: $R^{2}=0.853$ and $R^{2}(\operatorname{adj})=0.641$. Dependent variables are overall efficiency scores of each province. ${ }^{* * *},{ }^{* *}$, and ${ }^{*}$ indicate the parameter estimate is significantly different from zero at $0.001,0.01$, and 0.05 , respectively.

EI, CDI, and SWDI have no significant relationship with the overall efficiency. Thus, for the government, to improve the overall environmental performance, more efforts should be made to reduce the emission of $\mathrm{SO}_{2}$ in the production subsystem and $\mathrm{SO}_{2}$ in the pollutant treatment subsystem.

\section{Conclusion}

The Chinese regional industry system has structure of twostage network. However, most published papers on environmental performance analysis ignored the internal structure of the regional industry system. In our study, the internal structure of the Chinese regional industry system is considered. In real production system, some subsystems in twostage systems are not of equal importance, and this kind of two-stage systems usually has the feature that one subsystem dominates the other. Thus, in the efficiency evaluation of such systems, the leader and follower relationship should be considered. This is the first paper which introduced Stackelberg game theory to the environmental efficiency evaluation of two-stage production systems. Thus, we evaluate the environmental performance of the Chinese regional industry system not only considering the internal structure of production system but also considering the non-cooperative relationship between subsystems. These two subsystems are production subsystem and pollutant treatment subsystem, respectively. The newly proposed non-cooperative two-stage
DEA approach is applied to the case of the Chinese regional industry system. Some interesting conclusions are drawn. To make our conclusions more accurate, lengthening the time span of the empirical study may be a direction of future studies.

\section{Data Availability}

The data of Chinese regional industry systems used to support the findings of this study have been released in China Statistical Yearbook 2010 published by the China National Bureau of Statistics in 2010. The data could be downloaded in the website of China National Bureau of Statistics.

\section{Conflicts of Interest}

The authors declare that they have no conflicts of interest.

\section{Acknowledgments}

This research was funded by the National Natural Science Foundation of China (nos. 71701111, 71701102, 71803097, and 71801142).

\section{References}

[1] L. Chen and G. Jia, "Environmental efficiency analysis of China's regional industry: a data envelopment analysis (DEA) based approach," Journal of Cleaner Production, vol. 142, pp. 846-853, 2017.

[2] Y.-C. Chang and N. Wang, "Environmental regulations and emissions trading in China," Energy Policy, vol. 38, no. 7, pp. 3356-3364, 2010.

[3] H. Shi and L. Zhang, "China's environmental governance of rapid industrialisation," Environmental Politics, vol. 15, no. 2, pp. 271-292, 2006.

[4] Y. Geng, M. Haight, and Q. Zhu, "Empirical analysis of ecoindustrial development in China," Sustainable Development, vol. 15, no. 2, pp. 121-133, 2007.

[5] J. Wu, P. Xia, Q. Zhu, and J. Chu, "Measuring environmental efficiency of thermoelectric power plants: a common equilibrium efficient frontier DEA approach with fixed-sum undesirable output," Annals of Operations Research, vol. 275, no. 2, pp. 731-749, 2019.

[6] Y. Chang, R. J. Ries, and Y. Wang, "The embodied energy and environmental emissions of construction projects in China: an economic input-output LCA model," Energy Policy, vol. 38, no. 11, pp. 6597-6603, 2010.

[7] W. Cooper, L. M. Seiford, and J. Zhu, "Handbook on data envelopment analysis," International Series in Operations Research \& Management Science, Springer, New York, NY, USA, 2004.

[8] A. Shabani, R. F. Saen, and S. M. R. Torabipour, "A new data envelopment analysis (DEA) model to select eco-efficient technologies in the presence of undesirable outputs," Clean Technologies and Environmental Policy, vol. 16, no. 3, pp. 513-525, 2014.

[9] M. Khodakarami, A. Shabani, and R. F. Saen, "A new look at measuring sustainability of industrial parks: a two-stage data envelopment analysis approach," Clean Technologies and Environmental Policy, vol. 16, no. 8, pp. 1577-1596, 2014. 
[10] C. Cao, "Measuring sustainable development efficiency of urban logistics industry," Mathematical Problems in Engineering, vol. 2018, Article ID 9187541, 9 pages, 2018.

[11] X. Shi, "Efficiency bounds for two-stage production systems," Mathematical Problems in Engineering, vol. 2018, Article ID 2917537, 9 pages, 2018.

[12] P. Zhou, B. W. Ang, and K. L. Poh, "A survey of data envelopment analysis in energy and environmental studies," European Journal of Operational Research, vol. 189, no. 1, pp. 1-18, 2008a.

[13] Z. Hua, Y. Bian, and L. Liang, "Eco-efficiency analysis of paper mills along the Huai river: an extended DEA approach," Omega, vol. 35, no. 5, pp. 578-587, 2007.

[14] P. Zhou, B. W. Ang, and K. L. Poh, "Measuring environmental performance under different environmental DEA technologies," Energy Economics, vol. 30, no. 1, pp. 1-14, 2008.

[15] A. Khoshroo, M. Izadikhah, and A. Emrouznejad, "Improving energy efficiency considering reduction of $\mathrm{CO}_{2}$ emission of turnip production: a novel data envelopment analysis model with undesirable output approach," Journal of Cleaner Production, vol. 187, pp. 605-615, 2018.

[16] S. Reinhard, C. A. Knox Lovell, and G. J. Thijssen, "Environmental efficiency with multiple environmentally detrimental variables; estimated with SFA and DEA," European Journal of Operational Research, vol. 121, no. 2, pp. 287-303, 2000.

[17] Y.-T. Chang, N. Zhang, D. Danao, and N. Zhang, "Environmental efficiency analysis of transportation system in China: a non-radial DEA approach," Energy Policy, vol. 58, pp. 277-283, 2013.

[18] J. Chen, M. Song, and L. Xu, "Evaluation of environmental efficiency in China using data envelopment analysis," Ecological Indicators, vol. 52, pp. 577-583, 2015.

[19] R. Färe, S. Grosskopf, D.-W. Noh, and W. Weber, "Characteristics of a polluting technology: theory and practice," Journal of Econometrics, vol. 126, no. 2, pp. 469-492, 2005.

[20] Y. Han, C. Long, Z. Geng, and K. Zhang, "Carbon emission analysis and evaluation of industrial departments in China: an improved environmental DEA cross model based on information entropy," Journal of Environmental Management, vol. 205, pp. 298-307, 2018.

[21] G.-B. Bi, W. Song, P. Zhou, and L. Liang, "Does environmental regulation affect energy efficiency in China's thermal power generation? Empirical evidence from a slacks-based DEA model," Energy Policy, vol. 66, pp. 537-546, 2014.

[22] F. Wu, L. W. Fan, P. Zhou, and D. Q. Zhou, "Industrial energy efficiency with $\mathrm{CO}_{2}$ emissions in China: a nonparametric analysis," Energy Policy, vol. 49, pp. 164-172, 2012.

[23] Y. Chen, W. D. Cook, N. Li, and J. Zhu, "Additive efficiency decomposition in two-stage DEA," European Journal of Operational Research, vol. 196, no. 3, pp. 1170-1176, 2009.

[24] Z. Yang, "A two-stage DEA model to evaluate the overall performance of Canadian life and health insurance companies," Mathematical and Computer Modelling, vol. 43, no. 7-8, pp. 910-919, 2006.

[25] C.-W. Huang, F. N. Ho, and Y.-H. Chiu, "Measurement of tourist hotels' productive efficiency, occupancy, and catering service effectiveness using a modified two-stage DEA model in Taiwan," Omega, vol. 48, pp. 49-59. In press, 2014.

[26] J. F. Chu, J. Wu, C. Chu, and T. Zhang, "DEA-based fixed cost allocation in two-stage systems: leader-follower and satisfaction degree bargaining game approaches," Omega, 2019, In press.

[27] R. Banker, R. Natarajan, and D. Zhang, "Two-stage estimation of the impact of contextual variables in stochastic frontier production function models using Data Envelopment Analysis: second stage OLS versus bootstrap approaches," European Journal of Operational Research, vol. 278, no. 2, pp. 368-384, 2019.

[28] H. Li, C. Chen, W. D. Cook, J. Zhang, and J. Zhu, "Two-stage network DEA: who is the leader?," Omega, vol. 74, pp. 15-19, 2018.

[29] M. Song, S. Wang, and W. Liu, "A two-stage DEA approach for environmental efficiency measurement," Environmental Monitoring and Assessment, vol. 186, no. 5, pp. 3041-3051, 2014.

[30] J. Wu, B. B. Xiong, Q. X. An, J. S. Sun, and H. Q. Wu, "Totalfactor energy efficiency evaluation of Chinese industry by using two-stage DEA model with shared inputs," Annuals of Operations Research, vol. 255, no. 1-2, pp. 257-276, 2017.

[31] S. Lozano, "Technical and environmental efficiency of a twostage production and abatement system," Annuals of Operations Research, vol. 255, no. 1-2, pp. 199-219, 2017.

[32] W. B. Liu, Z. B. Zhou, C. Q. Ma, D. B. Liu, and W. F. Shen, "Two-stage DEA models with undesirable input-intermediateoutputs,” Omega, vol. 56, pp. 74-87, 2015.

[33] L. Liang, W. D. Cook, and J. Zhu, "DEA models for two-stage processes: game approach and efficiency decomposition," Naval Research Logistics, vol. 55, no. 7, pp. 643-653, 2008.

[34] Y. Zha and L. Liang, "Two-stage cooperation model with input freely distributed among the stages," European Journal of Operational Research, vol. 205, no. 2, pp. 332-338, 2010.

[35] M. Tavana and K. Khalili-Damghani, "A new two-stage Stackelberg fuzzy data envelopment analysis model," Measurement, vol. 53, pp. 277-296, 2014.

[36] L. M. Seiford and J. Zhu, "Modeling undesirable factors in efficiency evaluation," European Journal of Operational Research, vol. 142, no. 1, pp. 16-20, 2002.

[37] A. Charnes, W. W. Cooper, and E. Rhodes, "Measuring the efficiency of decision making units," European Journal of Operational Research, vol. 2, no. 6, pp. 429-444, 1978.

[38] Y. Bian and F. Yang, "Resource and environment efficiency analysis of provinces in China: a DEA approach based on Shannon's entropy," Energy Policy, vol. 38, no. 4, pp. 1909-1917, 2010. 


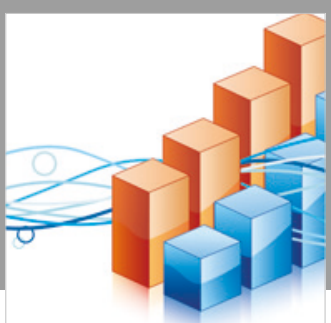

Advances in

Operations Research

\section{-n-m}
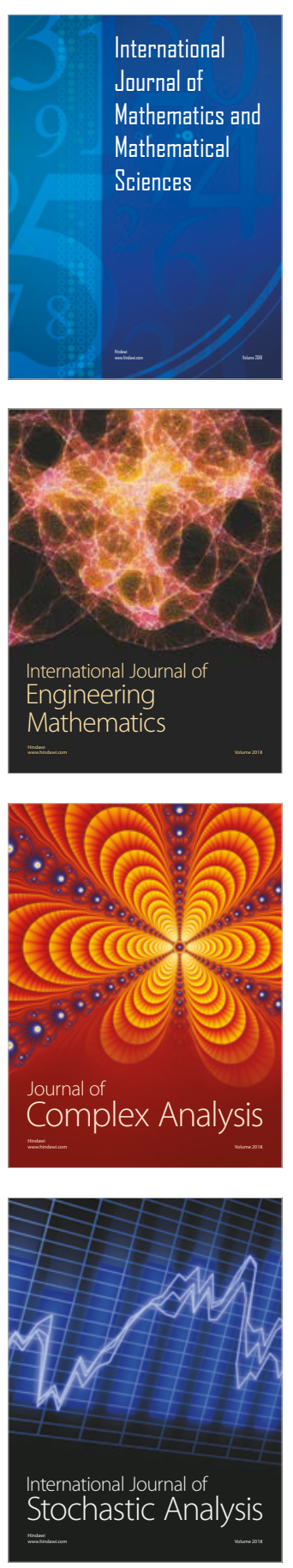
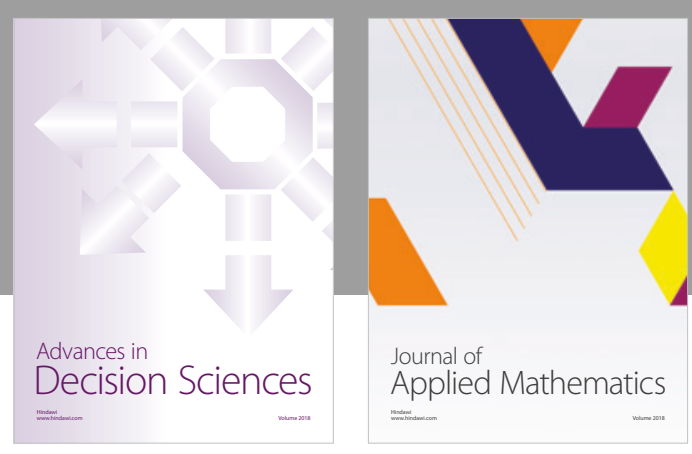

Journal of

Applied Mathematics
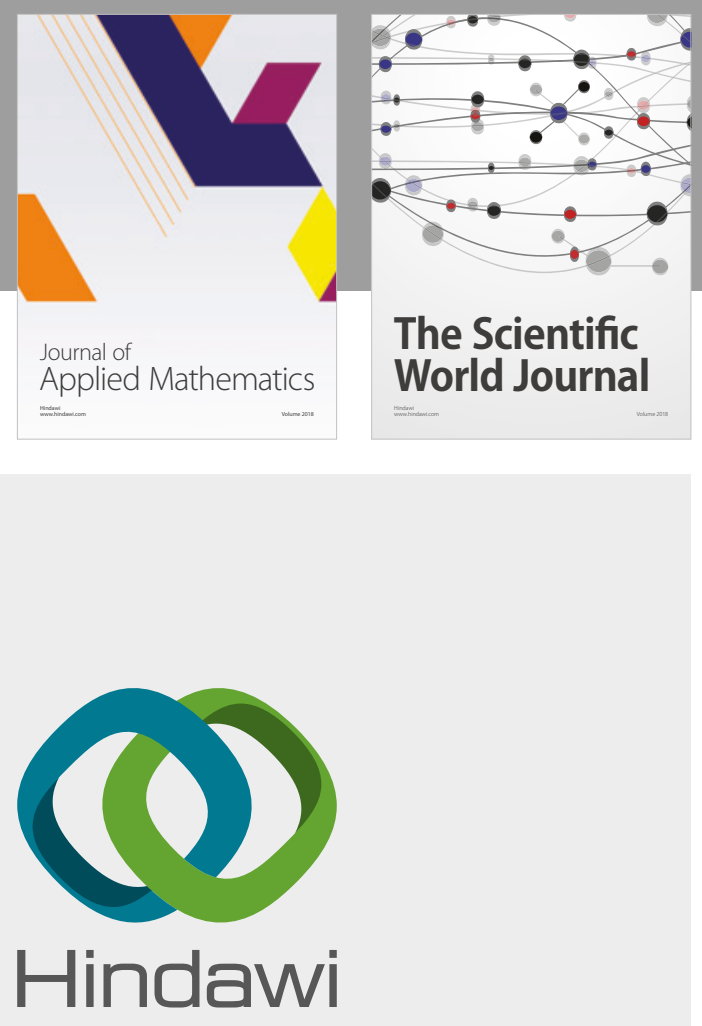

Submit your manuscripts at

www.hindawi.com

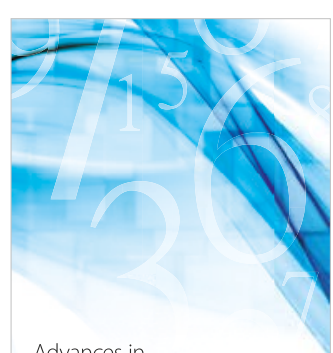

Advances in
Numerical Analysis
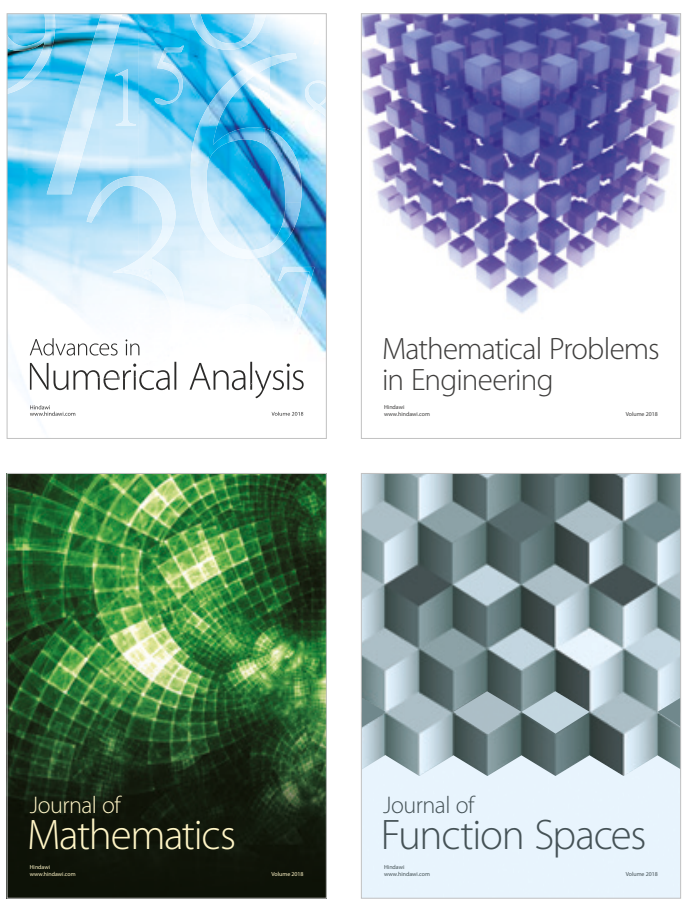

Mathematical Problems in Engineering

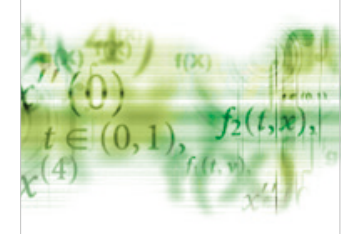

International Journal of

Differential Equations

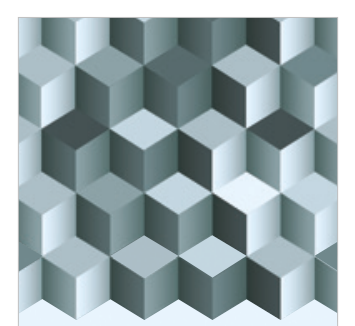

Journal of

Function Spaces

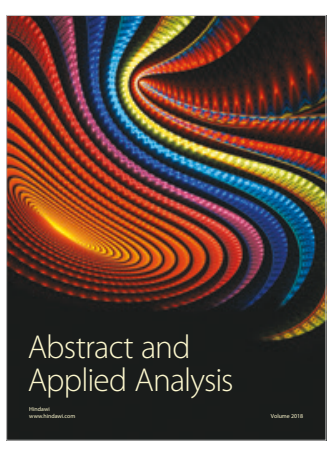

The Scientific

World Journal

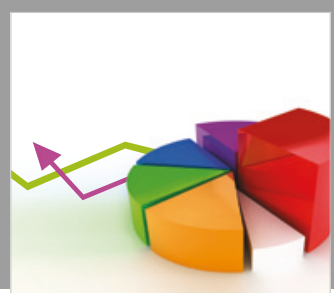

Journal of

Probability and Statistics
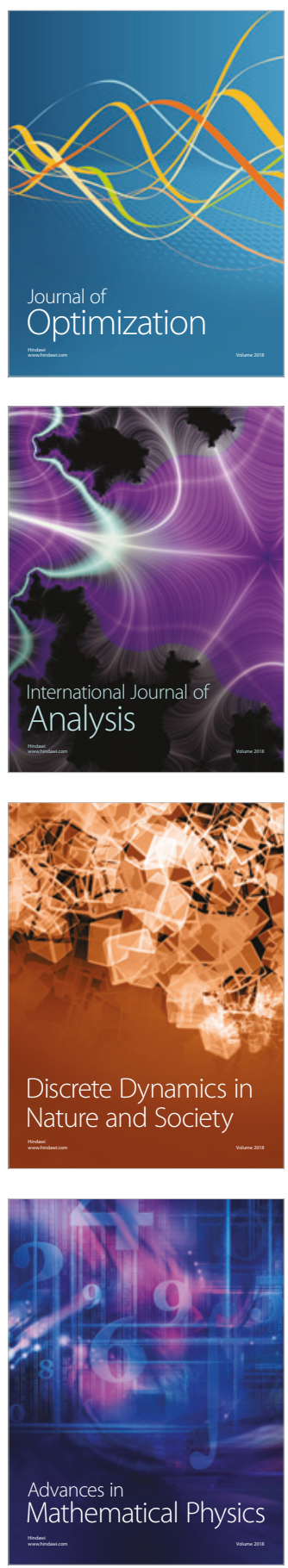